\title{
Mechatronics Application in Precision Sowing: A Review
}

\author{
Prem Veer Gautam ${ }^{1}$, H.L. Kushwaha ${ }^{2}$, Adarsh Kumar ${ }^{2}$ and Dilip Kumar Kushwaha ${ }^{2}$ \\ ${ }^{1}$ ICAR-CIAE, NabiBagh, Bhopal-462038, India \\ ${ }^{2}$ Division of Agricultural Engineering, IARI, New Delhi, India
}

*Corresponding author

\section{A B S T R A C T}

Keywords

Precision agriculture, Mechatronics, Electronics, Computing system, planter

Article Info

Accepted:

15 March 2019

Available Online:

10 April 2019
In agriculture profession high output is demanded with limited resources and machines are the costlier investment to the agriculture for a marginal and poor farmer. The accuracy and precision of an agricultural machine can be increased using the electronics and computing systems however it increases the cost of the machines. In spite of costlier investments, many researchers have been conducted the study on mechatronics application in precise sowing operation in recent years. Mechanical planters have problems of ground wheel skidding and vibration in the mechanical transmission that affect the hill to hill spacing. From the review, it was concluded that the mechatronic driving system performed better than mechanical driving system in both tilled and non-tilled fields. The increase in uniformity of seed placement was also reported. Thus, mechatronics based seed placement technique was found a better way to achieve accurate seed spacing with higher efficiency in planting. This paper reviews on the mechatronics and its application in precision planting.

\section{Introduction}

There are different methods such as broadcasting, dibbling, sowing behind country plough, seed drill and pneumatic planting for seed placement. Out of these methods, seed drill and pneumatic planting methods are more precise. Seed singulation capability of pneumatic planter is higher however it requires more energy. Seed drilling is most common method for cereals and pulse crops. In seed drill different type of metering mechanism are used for seed singulation. The seed drills are modified with seed metering mechanism for more precise singulation. These machines are called planter. Different types of seed mechanism are used for singulation in planter. These are variable orifice, fluted roller, internal double run, inclined plate and cup feed type. These mechanisms generally require ground wheel for transmission of the power. Due to load on ground wheel for power transmission to metering mechanism, the ground wheel skidding is observed. This skidding is responsible for non-uniform seed placement. Nowadays, agriculture requires new monitoring and control equipment and 
embedded systems for agricultural tractors and implements. The concept of intelligent instruments is one of the key reasons for this trend: instruments with embedded microprocessors providing the capabilities of self-calibration, self-diagnosis and local analog-to-digital and digital-to-analog conversion. The digital transmission of data also increases reliability due to automatic error detection and correction. These distributed systems are composed of several devices like sensors, actuators, control elements and supervision and control units, all of them intercommunicating in real time. To overcome ground wheel skidding, research on electronics based seed metering mechanism in planters and seed drills has been done for precise placement of seeds during planting.

Further drawbacks of the mechanical metering devices are the bulkiness of the system and vibrations that are induced on the planter or seed drill as it travels through the field. The final drawback of the mechanically driven system is the lack of communication of seed placement between row units on an implement. On current metering designs, the system has control over only the seeding population, but not the actual timing and placement of the seed. This means a seed is dropping into the furrow at a constant rate, but the row unit cannot detect the time and place where a seed is being placed relative to its neighbouring row units. Therefore, electronically controlled seed singulation devices can address many of the inefficiencies experienced in a mechanically driven seed metering device and have the potential to increase productivity and yield rates dramatically.

\section{Working principle and components of mechatronics sowing system}

The mechatronics mechanism works on the principle that shaft encoder senses the forward speed and transmit signals in the digital code to the microcontroller. The microcontroller synchronizes the forward speed of operation into 1:1 transmission ratio with the metering mechanism plate. The microcontroller transmits signals to motor driver module and driver connected to electric motor which rotate seed plate of the metering mechanism (Fig. 1).

He et al., (2017) designed a mechatronic system for four row planter consists of seed box, touch screen display, shaft encoder to measure travel speed, electric motors, seed meters, and power supply (Fig. 2). A twelve volt tractor battery provides power for the Mechatronics system. The value of travel speed is measured by an incremental encoder that is mounted on the shaft of a ground wheel. With the rotation of the ground wheel, the encoder outputs corresponding pulses from which the controller can calculate travel speed by measuring the number of pulses received within a given time. The drive motors are brushless DC motors, each with three Hall-effect sensors mounted in the back for measuring the positions of the $\mathrm{U}, \mathrm{V}$, and $\mathrm{W}$ rotors, which realizes current switching for the rotors. Simultaneously, the three Halleffect sensors measure the motor speed in real time to achieve closed loop control. A touch screen display allows the entry of parameters such as seed spacing, wheel slip ratio, diameter of the ground wheel, and number of seed holes per disk, displays travel speed, and seed plate rotation speed, and sounds alarms to warn of system malfunction. The controller main functionality is to output a pulse signal with a given frequency and duty cycle to control seed plate rotation speed based on travel speed to achieve uniform seed spacing.

\section{Trends of mechatronics in sowing}

As one of the trends of development on automation and intelligence of agricultural machinery in the $21^{\text {st }}$ century, all kinds of agricultural robots have been researched and 
developed to implement a number of agricultural production in many countries, such as picking, harvesting, weeding, pruning, planting, grafting, agricultural classification, etc. Application of electronics in agriculture has come with the technological advancement. The microcontroller or microprocessors for control of electronic circuitry are now economical and powerful tool with very low error margin as well as testing whether any machine operates in the right adjustment or not.

Not only the hardware but also open source user friendly software has been developed. This has encouraged researcher for specific electronics application in agriculture. The microcontroller can be used as per the need for actuation and sensing. The sensors are used by the researchers for seed placement for depth and distance control (Panning et al., 2000; Lan et al., 1999). Using electronics for metering mechanism may be one of the options to achieve accurate seed spacing with higher efficiency. Sensors can be used in precision planting if integrated with seed metering mechanism. A summary of mechatronics studies in seed sowing focusing on many different aspects is presented below in Table 1, 2, 3, 4 and 5.

\section{Performance parameters related to precision planter}

The sowing uniformity of seed distribution along the length of the row was analysed using the methods described by Kachman and Smith. Miss index (MI) is the percentage of seed spacings that are greater than 1.5 times the nominal seed spacing and indicates the percentage of missed seed locations or skips. Quality of feeding index(QFI) is the percentage of seed spacings that are more than half but no more than 1.5 times the nominal spacing and indicates the percentages of single seed drops. Precision index (PREC) is the coefficient of variation of the spacings (length) between the nearest seeds in a row that are classified as singles after omitting the outliers consisting of missing-seedings and multiples. The calculation formulas for MI, QFI and PREC (Gautam, 2017) are as follows:

$Q F I=\frac{n^{\prime}}{N}$
$M I=\frac{n^{\prime \prime}}{N}$

PREC $=\frac{S}{\bar{x}}$

$M U L T=\frac{n}{N}$

Where,

$\mathrm{S}=\sqrt{\frac{1}{N} \sum_{i=1}^{N}\left(x_{\tilde{i}}-\bar{x}\right)^{2}}$ (standard deviation of seed spacings)

$\bar{x}=\frac{1}{N} \sum_{i=1}^{N} x_{i}$

$x_{i}=$ is the $\mathrm{n}^{\text {th }}$ seed spacing

$\mathrm{N}=$ Total number of seed spacings, and $n^{\prime \prime}=$ Number of spacings in the region greater than 1.5 times of the theoretical spacing $n^{\prime}=$ Number of spacings between 0.5 times the theoretical spacing and 1.5 times of the theoretical spacing $\mathrm{n}=$ Number of spacing's in the region less than or equal to 0.5 times of the theoretical spacing

Suggested upper limit of PREC index for single seed planter is $29 \%$ (Nejadi and Raoufat, 2013; Raoufat and Mahmoodieh, 2005). Planting performance indicators were evaluated by using the criteria provided in Table 6 (Aykas et al., 2013; ISO 7256/11984(E) Standard, 1984; Önal et al., 2012). 
Table.1 Mechatronics in seed drill

\begin{tabular}{|c|c|c|c|c|c|c|c|}
\hline $\mathrm{S} / \mathrm{N}$ & Researcher Name & Seed & Research Topic & Electronic Components & Source & Address of Researcher & Journal Name \\
\hline 1. & $\begin{array}{l}\text { M. Jafari, A. Hemmat and M. } \\
\text { Sadeghi }\end{array}$ & Wheat & $\begin{array}{l}\text { Development and } \\
\text { Performance Assessment } \\
\text { of a DC Electric Variable- } \\
\text { Rate Controller for Use on } \\
\text { Grain Drills. }\end{array}$ & $\begin{array}{l}\text { DC motor, encoders(E50S-2500-3-2-24 } \\
\text { and ISE-200-5V)), GPS receiver, pulse- } \\
\text { with-modulation (PWM) DC motor } \\
\text { controller, laptop, 12-24V supply, 12-step } \\
\text { CMOS4040 IC, A 74LS138 IC, PID } \\
\text { controller, MOSFET, 74LS373 IC }\end{array}$ & $\begin{array}{l}\text { Jafari et al., } \\
(2010)\end{array}$ & $\begin{array}{l}\text { Department of Farm Machinery, College } \\
\text { of Agriculture, Isfahan University of } \\
\text { Technology, Isfahan 84156-83111, Iran. } \\
\text { ahemmat@cc.iut.ac.ir }\end{array}$ & $\begin{array}{l}\text { Computers and } \\
\text { Electronics in } \\
\text { Agriculture }\end{array}$ \\
\hline 2. & $\begin{array}{l}\text { Caner Cuhac, } \\
\text { ReinoVirrankoski, Petri } \\
\text { Hanninen, Mohammed } \\
\text { Elmusrati, HermanniHoopakka } \\
\text { and HeikkiPalomaki }\end{array}$ & $\begin{array}{l}\text { Rye, Wheat, } \\
\text { Barley, } \\
\text { Corn and } \\
\text { Oat }\end{array}$ & $\begin{array}{l}\text { Seed Flow Monitoring in } \\
\text { Wireless Sensor Networks. }\end{array}$ & $\begin{array}{l}\text { LED, Light Dependent Resistors (LDR), } \\
\text { receiver UWASA Node, ultrasonic and } \\
\text { microwave sensor, SURFbuttons, SPI } \\
\text { bridge, LCD display, transmitter, battery }\end{array}$ & $\begin{array}{l}\text { Cuhac et al., } \\
(2012)\end{array}$ & $\begin{array}{l}\text { University of Vaasa } \\
\text { Department of Computer Science } \\
\text { Communications and Systems } \\
\text { Engineering Group P.O.Box 700, FI- } \\
65101 \text { Vaasa, Finland }\end{array}$ & $\begin{array}{l}\text { Workshop on } \\
\text { Wireless Sensor } \\
\text { Systems }\end{array}$ \\
\hline 3. & $\begin{array}{l}\text { HifjurRaheman and Rajeev } \\
\text { Kumar }\end{array}$ & $\begin{array}{l}\text { Wheat and } \\
\text { Ragi }\end{array}$ & $\begin{array}{l}\text { An Embedded System for } \\
\text { Detecting Seed Flow in the } \\
\text { Delivery Tube of a Seed } \\
\text { Drill }\end{array}$ & $\begin{array}{l}12 \mathrm{~V} \text { DC battery, DC motor, } \\
\text { potentiometer, DC motor driver, } \\
\text { microcontroller (Arduinouno), IR sensor } \\
\text { and buzzer }\end{array}$ & $\begin{array}{l}\text { Raheman\& } \\
\text { Kumar, (2015) }\end{array}$ & $\begin{array}{l}\text { Agricultural and Food Engineering } \\
\text { Department, IIT, Kharagpur, India }\end{array}$ & $\begin{array}{l}\text { Proceeding of } \\
\text { International } \\
\text { Conference on } \\
\text { ACBEE }\end{array}$ \\
\hline 4. & $\begin{array}{l}\text { S. Kamgar, F. Noei-Khodabadi } \\
\text { and S.M. Shafaei }\end{array}$ & Wheat & $\begin{array}{l}\text { Design, Development and } \\
\text { Field Assessment of a } \\
\text { Controlled Seed Metering } \\
\text { Unit to be Used in Grain } \\
\text { Drills for Direct Seeding of } \\
\text { Wheat }\end{array}$ & $\begin{array}{l}\text { digital encoder (Autonics E50S8-1000), } \\
\text { variable-rate DCM (model: D12-8001- } \\
45 \mathrm{~W}), 4 \times 4 \text { matrix keyboard Device, } \\
\text { Atmega } 16 \text { microcontroller, } 16 \times 2 \mathrm{LCD} \text {, } \\
\text { PATA cable, PID controller, direct current } \\
\text { voltage transducer }\end{array}$ & $\begin{array}{l}\text { Kamgar et al., } \\
(2015)\end{array}$ & $\begin{array}{l}\text { Department of Biosystems Engineering, } \\
\text { College of Agriculture, Shiraz } \\
\text { University, Shiraz 71441-65186, Iran. } \\
\text { smshafaei@ shirazu.ac.ir }\end{array}$ & $\begin{array}{l}\text { Information } \\
\text { Processing in } \\
\text { Agriculture }\end{array}$ \\
\hline 5. & $\begin{array}{l}\text { SørenKirkegaard Nielsen, Lars } \\
\text { JuhlMunkholm, Mathieu } \\
\text { Lamandé, Michael Nørremark, } \\
\text { Gareth T.C. Edwards and Ole } \\
\text { Green }\end{array}$ & $\begin{array}{l}\text { Spring } \\
\text { Barley }\end{array}$ & $\begin{array}{l}\text { Seed Drill Depth Control } \\
\text { System for Precision } \\
\text { Seeding }\end{array}$ & $\begin{array}{l}\text { Linear position sensors TX2, P43 } \\
\text { ultrasonic height sensors, ultrasonic } \\
\text { sensors, X20 controller, electrohydraulic } \\
4 / 3 \text { oil direction valve SV08-47B, GNSS } \\
\text { BT-Q1000XT and pilot-controlled leak- } \\
\text { proof }\end{array}$ & $\begin{array}{l}\text { Nielsen et al., } \\
\text { (2018) }\end{array}$ & $\begin{array}{l}\text { Aarhus University, Faculty of Science } \\
\text { and Technology, Department of } \\
\text { Engineering, Denmark }\end{array}$ & $\begin{array}{l}\text { Computers and } \\
\text { Electronics in } \\
\text { Agriculture }\end{array}$ \\
\hline 6. & $\begin{array}{l}\text { Karan Singh, K. N. Agrawal } \\
\text { and Anurag Kumar Dubey }\end{array}$ & Soyabean & $\begin{array}{l}\text { Development of the } \\
\text { Contoller based Seed cum } \\
\text { Fertilizer Drill }\end{array}$ & $\begin{array}{l}\text { Programmable PLC/PMW controller, } \\
\text { inductive proximity sensor, AC motor, } \\
24 V \text { DC motor, SMPS, encoder, USB, RS- } \\
232 \text { and flash card }\end{array}$ & $\begin{array}{l}\text { Singh et al., } \\
(2012)\end{array}$ & $\begin{array}{l}\text { ICAR-CIAE, Bhopal, India. } \\
\text { ksingh@ ciae.res.in }\end{array}$ & $\begin{array}{l}12 \text { th } \\
\text { International } \\
\text { Conference on } \\
\text { IEEE. }\end{array}$ \\
\hline 7. & $\begin{array}{l}\text { HadiKarimi, HosseinNavid and } \\
\text { AsgharMahmoudi }\end{array}$ & $\begin{array}{l}\text { Wheat, corn } \\
\text { and pelleted } \\
\text { tomato }\end{array}$ & $\begin{array}{l}\text { Online laboratory } \\
\text { evaluation of seeding- } \\
\text { machine application by an } \\
\text { acoustic technique }\end{array}$ & $\begin{array}{l}\text { Microphone (VM-034CY), sound card } \\
\text { (Intel『 } 82801 \text { BA/BAM AC'97 Audio } \\
\text { controller), MATLAB software }\end{array}$ & $\begin{array}{l}\text { Karimi et al., } \\
(2015)\end{array}$ & $\begin{array}{l}\text { University of Tabriz, Faculty of } \\
\text { Agriculture, Department of Agricultural } \\
\text { Machinery. Tabriz, Iran } \\
\text { hadiekarimi@gmail.com }\end{array}$ & $\begin{array}{l}\text { Spanish Journal } \\
\text { of Agricultural } \\
\text { Research }\end{array}$ \\
\hline
\end{tabular}


Table.2 Mechatronics application in mechanical planter

\begin{tabular}{|c|c|c|c|c|c|c|c|}
\hline $\mathrm{S} / \mathrm{N}$ & Researcher Name & Seed & Research Topic & Electronic Components & Source & Address of Researcher & Journal Name \\
\hline 8. & $\begin{array}{l}\text { D. E. Wilkins and D. H. } \\
\text { Lenker }\end{array}$ & Lettuce & $\begin{array}{l}\text { A microprocessor- } \\
\text { controlled planter }\end{array}$ & $\begin{array}{l}\text { 8080Amicroprocessor, LED, } \\
\text { phototransistor, power source and solenoid } \\
\text { air valve }\end{array}$ & $\begin{array}{l}\text { Wilkins and } \\
\text { Lenker, (1981) }\end{array}$ & $\begin{array}{l}\text { USDA SEA-AR, Columbia Plateau } \\
\text { Conservation Research Centre, } \\
\text { Pendleton(US) }\end{array}$ & $\begin{array}{l}\text { Transactions of } \\
\text { the ASAE }\end{array}$ \\
\hline 9. & $\begin{array}{l}\text { P. R.Shinde, A. B. Lende, } \\
\text { S.V. Rane, S. } \\
\text { A.Nawale, M. } \\
\text { S. Patwardhan, and L. } \\
\text { V.Gharate }\end{array}$ & Groundnut & $\begin{array}{l}\text { Development and Functional } \\
\text { Test of Electronic Metering } \\
\text { Mechanism for Bullock Drawn } \\
\text { JyotiMulticrop Planter. }\end{array}$ & $\begin{array}{l}\text { Opto-isolator sensors, microcontroller } \\
\text { (ATMEL89), BC547 and SL100 } \\
\text { transistors, 2x16 LCD, solenoid switches, } \\
\text { 12Vbattery and keyboard. }\end{array}$ & $\begin{array}{l}\text { Shinde et al., } \\
\text { (2009) }\end{array}$ & $\begin{array}{l}\text { Department of Farm Machinery and } \\
\text { Power, Dr. A. S. College of Agricultural } \\
\text { Engineering, Mahatma } \\
\text { PhuleKrishiVidyapeeth, Rahuri - } 413 \\
\text { 722, India. }\end{array}$ & $\begin{array}{l}\text { International } \\
\text { Journal of } \\
\text { Agriculture } \\
\text { Environment and } \\
\text { Biotechnology }\end{array}$ \\
\hline 10. & $\begin{array}{l}\text { Lianming Xia, Xiangyou } \\
\text { Wang, DuanyangGeng and } \\
\text { Qingfeng Zhang }\end{array}$ & $\begin{array}{l}\text { Maize and } \\
\text { wheat }\end{array}$ & $\begin{array}{l}\text { Performance Monitoring } \\
\text { System for Precision Planter } \\
\text { Based onMSP430-CT171 }\end{array}$ & $\begin{array}{l}\text { IR LED, phototransistor, photoelectric } \\
\text { sensor, LM339, microcontroller (MSP430- } \\
\text { CT171), display module } \\
\text { (LMC240128ZK), LCD, Bluetooth } \\
\text { module, FS-BT485A serial adapter, } \\
\text { RS232, Buzzer, Stepper motor, motor } \\
\text { driver, keyboard circuit and power driver }\end{array}$ & Xia et al., (2010) & $\begin{array}{l}\text { School of Agricultural and Food } \\
\text { Engineering, Shandong University of } \\
\text { Technology Zibo, Shandong, China }\end{array}$ & $\begin{array}{l}\text { International } \\
\text { Conference on } \\
\text { Computer and } \\
\text { Computing } \\
\text { Technologies in } \\
\text { Agriculture }\end{array}$ \\
\hline 11. & $\begin{array}{l}\text { M. Anantachara, Prasanna } \\
\text { G.V. Kumar and T. } \\
\text { Guruswamya }\end{array}$ & Peanut & $\begin{array}{l}\text { NN Prediction of Performance } \\
\text { Parameters of an Inclined Plate } \\
\text { Seed Metering Device and Its } \\
\text { Reverse Mapping for the } \\
\text { Determination of Optimum } \\
\text { Design and Operational } \\
\text { Parameters }\end{array}$ & $\begin{array}{l}\text { Opto-electronic seed counter, electric } \\
\text { motor, ANN models }\end{array}$ & $\begin{array}{l}\text { Anantachara et } \\
\text { al., (2010) }\end{array}$ & $\begin{array}{l}\text { Department of Farm Machinery, College } \\
\text { of Agricultural Engineering, University } \\
\text { of Agricultural Sciences, Raichur } \\
584101, \text { Karnataka, India } \\
\text { gvpk@yahoo.com }\end{array}$ & $\begin{array}{l}\text { Computers and } \\
\text { Electronics in } \\
\text { Agriculture }\end{array}$ \\
\hline 12. & $\begin{array}{l}\text { O. Hajahmed, E. Tola, K. } \\
\text { A. Al-Gaadi and A. F. } \\
\text { Kheiralla }\end{array}$ & $\begin{array}{l}\text { Chickpeas } \\
\text { seeds }\end{array}$ & $\begin{array}{l}\text { Development of an Opto- } \\
\text { Electronic Monitoring System } \\
\text { for Crop Planter Seed Metering } \\
\text { Unit }\end{array}$ & $\begin{array}{l}\text { AC motor ( } 220 \text { Volt, } 0.4 \mathrm{~kW}) \text {, Digital } \\
\text { Fiber Sensor (E3X-DA-S), rotary encoder } \\
\text { (E6B2- CWZ6C), microcontroller (Atmel } \\
\text { ATMega16L) }\end{array}$ & $\begin{array}{l}\text { Hajahmed et al., } \\
\text { (2011) }\end{array}$ & $\begin{array}{l}\text { Precision Agriculture Research Chair } \\
\text { (PARC), College of Food and } \\
\text { Agricultural Sciences, King Saud } \\
\text { University. P.O. Box 2460, Riyadh } \\
\text { 11451, Saudi Arabia }\end{array}$ & $\begin{array}{l}\text { Middle-East } \\
\text { Journal of } \\
\text { Scientific } \\
\text { Research }\end{array}$ \\
\hline 13. & $\begin{array}{l}\text { T. P. Singh and } \\
\text { D. M. Mane }\end{array}$ & Okra & $\begin{array}{l}\text { Development and Laboratory } \\
\text { Performance of an } \\
\text { Electronically Controlled } \\
\text { Metering Mechanism for Okra } \\
\text { Seed }\end{array}$ & $\begin{array}{l}\text { Proximity sensor, pulse generator, BCD } \\
\text { counter(IC } 4510 \text { ), Timer (IC } 4093 \text { ), Relay } \\
\text { unit, DC motor, } 12 \mathrm{~V} \text { tractor battery, screw } \\
\text { control knob. }\end{array}$ & $\begin{array}{l}\text { Singh and Mane, } \\
\text { (2011) }\end{array}$ & $\begin{array}{l}\text { Farm machinery and Power Engineering } \\
\text { College of Technology G. B. Pant } \\
\text { University of Agriculture and } \\
\text { Technology Pantnagar-263145, } \\
\text { Uttarakhand INDIA. } \\
\text { tpsingh_62@yahoo.co.in }\end{array}$ & $\begin{array}{l}\text { Agricultural } \\
\text { Mechanization in } \\
\text { Asia, Africa, and } \\
\text { Latin America }\end{array}$ \\
\hline 14. & $\begin{array}{l}\text { H. Navid, S. Ebrahimian, } \\
\text { H. R. Gassemzadeh and M. } \\
\text { J. Mousavinia }\end{array}$ & $\begin{array}{l}\text { Pelleted } \\
\text { tomato } \\
\text { seeds }\end{array}$ & $\begin{array}{l}\text { Laboratory Evaluation of Seed } \\
\text { Metering Device using Image } \\
\text { Processing Method }\end{array}$ & $\begin{array}{l}\text { Digital camera (Nikon, D70), USB port } \\
\text { and MATLAB software }\end{array}$ & $\begin{array}{l}\text { Navid et al., } \\
\text { (2011) }\end{array}$ & $\begin{array}{l}\text { Department of Agricultural Machinery } \\
\text { Engineering, University of Tabriz, } \\
\text { Tabriz, I.R. Iran navid@tabrizu.ac.ir }\end{array}$ & $\begin{array}{l}\text { Australian Journal } \\
\text { of Agricultural } \\
\text { Engineering }\end{array}$ \\
\hline 15. & $\begin{array}{l}\text { TejminderKaur and Dilip } \\
\text { Kumar }\end{array}$ & Wheat & $\begin{array}{l}\text { Design and Development of } \\
\text { Calibration Unit for Precision } \\
\text { Planter. }\end{array}$ & $\begin{array}{l}\text { Frame light barrier sensor, Proximity } \\
\text { sensor (gear tooth sensor), SMU, } 1 \text { hp AC } \\
\text { motor, Yaskawa J1000 AC drive, RS } 232 \\
\text { and USB communication }\end{array}$ & $\begin{array}{l}\text { Kaur\& Kumar, } \\
\text { (2013) }\end{array}$ & $\begin{array}{l}\text { Centre for Development of Advanced } \\
\text { Computing(C-DAC), Mohali, India }\end{array}$ & $\begin{array}{l}\text { International } \\
\text { Journal of } \\
\text { Computer } \\
\text { Science, } \\
\text { Engineering and } \\
\text { Applications }\end{array}$ \\
\hline
\end{tabular}




\begin{tabular}{|c|c|c|c|c|c|c|c|}
\hline 16. & $\begin{array}{l}\text { Du Ruicheng, Gong } \\
\text { Bingcai, Liu Ningning, } \\
\text { Wang Chenchen, Yang } \\
\text { Zidong and Ma Mingjian }\end{array}$ & Corn & $\begin{array}{l}\text { Design and Experiment on } \\
\text { Intelligent Fuzzy Monitoring } \\
\text { System for Corn Planters }\end{array}$ & $\begin{array}{l}\text { On-board computers, GPS receivers, } \\
\text { digital cameras, tilt sensor, USB-CAN } \\
\text { interface module, displacement sensors, } \\
\text { electronically controlled stepless spacing } \\
\text { regulator, CAN bus analog input module, } \\
\text { CAN bus digital input and output modules, } \\
\text { CAN bus pulse counting module, seed } \\
\text { tank sensor, fertilizer tank sensor, seeding } \\
\text { orifice sensor and gear speed sensor }\end{array}$ & $\begin{array}{l}\text { Ruicheng et al., } \\
\text { (2013) }\end{array}$ & $\begin{array}{l}\text { School of Agriculture and Food } \\
\text { Engineering, Shandong University of } \\
\text { Technology, Zibo 255049, Shandong, } \\
\text { China }\end{array}$ & $\begin{array}{l}\text { International } \\
\text { Journal of } \\
\text { Agricultural and } \\
\text { Biological } \\
\text { Engineering }\end{array}$ \\
\hline 17. & $\begin{array}{l}\text { JavadTaghinezhad, Reza } \\
\text { Alimardani and Ali Jafari }\end{array}$ & Sugarcane & $\begin{array}{l}\text { Design a Capacitive Sensor for } \\
\text { Rapid Monitoring of Seed Rate } \\
\text { of Sugarcane Planter }\end{array}$ & $\begin{array}{l}\text { Rectangular parallel plate capacitor, } \\
\text { electronic circuitry, microcontroller, and } \\
\text { display unit }\end{array}$ & $\begin{array}{l}\text { Taghinezhad et } \\
\text { al., (2013) }\end{array}$ & $\begin{array}{l}\text { Department of Agricultural Machinery } \\
\text { Engineering, Faculty of Agricultural } \\
\text { Engineering and Technology University } \\
\text { of Tehran, Iran }\end{array}$ & $\begin{array}{l}\text { Agricultural } \\
\text { Engineering } \\
\text { International: } \\
\text { CIGR Journal }\end{array}$ \\
\hline 18. & $\begin{array}{l}\text { Margarita Velandia, } \\
\text { Michael Buschermohle, } \\
\text { James A. Larson, } \\
\text { Nathanael M. Thompson, } \\
\text { Brandon Michael Jernigan }\end{array}$ & $\begin{array}{l}\text { Corn, } \\
\text { soybean } \\
\text { and cotton }\end{array}$ & $\begin{array}{l}\text { The economics of Automatic } \\
\text { Section Control Technology for } \\
\text { Planters: A Case Study of } \\
\text { Middle and West Tennessee } \\
\text { Farms }\end{array}$ & $\begin{array}{l}\text { GPS receiver (Trimble EZ-Guide } 500 \\
\text { system), GPS antenna (Trimble AgGPS } 25 \\
\text { antenna), Intercom RTK Bridge cellular } \\
\text { modem, netbook computer, data logger } \\
\text { and switches }\end{array}$ & $\begin{array}{l}\text { Velandia et al., } \\
\text { (2013) }\end{array}$ & $\begin{array}{l}\text { Department of Agricultural and } \\
\text { Resource Economics, The University of } \\
\text { Tennessee, Knoxville, TN, United States }\end{array}$ & $\begin{array}{l}\text { Computers and } \\
\text { Electronics in } \\
\text { Agriculture }\end{array}$ \\
\hline 19. & $\begin{array}{l}\text { ZhaiJianbo, Xia Junfang, } \\
\text { Zhou Yong and Zhang } \\
\text { Shun }\end{array}$ & Soybean & $\begin{array}{l}\text { Design and Experimental Study } \\
\text { of the Control System for } \\
\text { Precision Seed-Metering } \\
\text { Device }\end{array}$ & $\begin{array}{l}\text { Hall sensor, AT89S51 single chip } \\
\text { microcomputer, Motor control module, } \\
\text { 57H76-03 stepper motor and adjustable } \\
\text { speed motor }\end{array}$ & $\begin{array}{l}\text { Jianbo et al., } \\
\text { (2014) }\end{array}$ & $\begin{array}{l}\text { College of Engineering, Huazhong } \\
\text { Agricultural University, Wuhan } 430070 \text {, } \\
\text { Hubei Province, China }\end{array}$ & IJABE \\
\hline 20. & $\begin{array}{l}\text { V. V. Aware and S. V. } \\
\text { Aware }\end{array}$ & Cowpea & $\begin{array}{l}\text { Development of } \\
\text { Microprocessor based } \\
\text { Electronic Metering } \\
\text { Mechanism for Seed-an } \\
\text { Approach }\end{array}$ & $\begin{array}{l}\text { Microcontroller (AT89C51 IC), inverter, } \\
\text { D.C. motor, operational amplifier } \\
\text { (LM741), } 2 \text { X } 16 \text { LCD Display, tactile } \\
\text { switches, } 12 \text { MHz crystal oscillator, } \\
\text { Capacitors, Diodes, opto- electric sensor, } \\
230 \text { V, } 50 \text { Hz A.C supply, Transformer }\end{array}$ & $\begin{array}{l}\text { Aware\& Aware, } \\
(2014)\end{array}$ & $\begin{array}{l}\text { Department of Farm Machinery and } \\
\text { Power, College of Agricultural } \\
\text { Engineering and Technology, Dr. B.S. } \\
\text { Konkan Krishi Vidyapeeth, Dapoli, } \\
\text { RATNAGIRI (M.S.), INDIA }\end{array}$ & $\begin{array}{l}\text { Engineering and } \\
\text { Technology in } \\
\text { India }\end{array}$ \\
\hline 21. & $\begin{array}{l}\text { CristianIacomi and } \\
\text { Octavian Popescu }\end{array}$ & $\begin{array}{l}\text { Pelleted } \\
\text { lettuce and } \\
\text { carrot }\end{array}$ & $\begin{array}{l}\text { A New Concept for Seed } \\
\text { Precision Planting }\end{array}$ & $\begin{array}{l}\text { Linear solenoid actuator, Optoelectronic } \\
\text { sensor (IR LED, phototransistor) and } \\
\text { electronic switch }\end{array}$ & $\begin{array}{l}\text { Iacomi\&Popescu, } \\
\text { (2015) }\end{array}$ & $\begin{array}{l}\text { University of Agronomic Sciences and } \\
\text { Veterinary Medicine of Bucharest, } 59 \\
\text { Marasti Blvd, 011464, Bucarest-1, } \\
\text { Romania }\end{array}$ & $\begin{array}{l}\text { Agriculture and } \\
\text { agricultural } \\
\text { science procedia }\end{array}$ \\
\hline 22. & $\begin{array}{l}\text { Niu Kang, Fang Xianfa, } \\
\text { Liu Yangchun, LüChengxu } \\
\text { and Yuan Yanwei }\end{array}$ & Potato & $\begin{array}{l}\text { Optimized Design and } \\
\text { Performance Evaluation of an } \\
\text { Electric Cup-Chain Potato } \\
\text { Metering Device }\end{array}$ & $\begin{array}{l}\text { Microcontroller (PIC18F2580), motor } \\
\text { control circuit, GPS, D/A translate box, } \\
\text { servo motor, differential GPS, RS232 } \\
\text { serial port, PID control algorithm, servo } \\
\text { motor (SGMJV-04ADE6S), servo driver } \\
\text { (SGDV-2R8A01B002000) analysis } \\
\text { software DPS v7.05 and Matlab R2012A }\end{array}$ & $\begin{array}{l}\text { Kang et al., } \\
(2017)\end{array}$ & $\begin{array}{l}\text { College of Engineering, China } \\
\text { Agricultural University, Beijing 100083, } \\
\text { China yyw215@163.com }\end{array}$ & $\begin{array}{l}\text { International } \\
\text { Journal of } \\
\text { Agricultural and } \\
\text { Biological } \\
\text { Engineering }\end{array}$ \\
\hline 23. & $\begin{array}{l}\text { ShankhaKoley, Y.C. Bhatt, } \\
\text { Gajendra Singh, Sunil } \\
\text { Joshi and H. K. Jain }\end{array}$ & Ground nut & $\begin{array}{l}\text { Development of Electronic } \\
\text { Metering Mechanism for } \\
\text { Precision Planting of Seeds }\end{array}$ & $\begin{array}{l}\text { Proximity sensor, microcontroller } \\
\text { (AT89C51), motor driver (L293D), DC } \\
\text { motor, potentiometer }(150 \mathrm{~W}) \text { and power } \\
\text { source }\end{array}$ & $\begin{array}{l}\text { Koley et al., } \\
\text { (2017) }\end{array}$ & $\begin{array}{l}\text { Indian Institute of Technology, } \\
\text { Kharagpur, India }\end{array}$ & $\begin{array}{l}\text { International } \\
\text { Journal of Current } \\
\text { Microbiology and } \\
\text { Applied Sciences }\end{array}$ \\
\hline
\end{tabular}


Table.3 Mechatronics in Pneumatic Planter

\begin{tabular}{|c|c|c|c|c|c|c|c|}
\hline $\mathrm{S} / \mathrm{N}$ & Researcher Name & Seed & Research Topic & Electronic Components & Source & Address of Researcher & Journal Name \\
\hline 24. & $\begin{array}{l}\text { Y. Lan, M. F. Kocher } \\
\text { and J. A. Smith }\end{array}$ & $\begin{array}{l}\text { Sugar beet and } \\
\text { pelleted } \\
\text { chicory }\end{array}$ & $\begin{array}{l}\text { Opto-electronic Sensor System } \\
\text { for Laboratory Measurement of } \\
\text { Planter Seed Spacing with Small } \\
\text { Seeds }\end{array}$ & $\begin{array}{l}\text { NIR LEDs (EG and G VACTEC GaAs } \\
\text { VTE3322LA), phototransistors (EG and G } \\
\text { VACTEC NPN VTT3323LA), digital } \\
\text { input/output (I/O) board, opto-electronic } \\
\text { sensor, Hall-effect switch ,3) } 58 \mathrm{MHz} \\
\text { clock, data-acquisition program }\end{array}$ & Lan et al., (1999) & $\begin{array}{l}\text { Department of Biological Systems } \\
\text { Engineering, University of Nebraska, } \\
\text { Lincoln, NE 68583, USA }\end{array}$ & $\begin{array}{l}\text { Journal of } \\
\text { Agricultural } \\
\text { Engineering } \\
\text { Research }\end{array}$ \\
\hline 25. & $\begin{array}{l}\text { ZelihaBereketBarut } \\
\text { and KadirYiğit }\end{array}$ & Corn & $\begin{array}{l}\text { Design of Electronic-Based } \\
\text { Measurement System for Seed } \\
\text { Spacing Measurement in } \\
\text { Precision Planters }\end{array}$ & $\begin{array}{l}\text { Microcontroller (AT89S8252), fiber-optic } \\
\text { sensor amplifier. } 2 \text { fiber-optic sensors and } \\
2 \text { mutual fiber-optic cables, analog-digital } \\
\text { converter, electrical motor. }\end{array}$ & $\begin{array}{l}\text { Barut\&Yiğit, } \\
(2008)\end{array}$ & $\begin{array}{l}\text { Çukurova University, Faculty of } \\
\text { Agriculture, Department of Agricultural } \\
\text { Machinery, 01330, Adana, Turkey }\end{array}$ & $\begin{array}{l}10^{\text {th }} \text { International } \\
\text { Congress on } \\
\text { Mechanization } \\
\text { and Energy in } \\
\text { Agriculture }\end{array}$ \\
\hline 26. & $\begin{array}{l}\text { Okan ÖNAL and } \\
\text { İsmet ÖNAL }\end{array}$ & $\begin{array}{l}\text { Hybrid maize } \\
\text { and cotton }\end{array}$ & $\begin{array}{l}\text { Development of a Computerized } \\
\text { Measurement System for In-Row } \\
\text { Seed Spacing Accuracy }\end{array}$ & $\begin{array}{l}\text { CMS hardware, laser pointer, notebook } \\
\text { computer, optical mouse (Microsoft } \\
\text { Optical Mouse } 3000 \text { ), USB cable } \\
\text { extension, Light meter (Lutron model Lx- } \\
1108 \text { ) and CMS software }\end{array}$ & $\begin{array}{l}\text { Önal \& Önal, } \\
\text { (2009) }\end{array}$ & $\begin{array}{l}\text { DokuzEylül University, Department of } \\
\text { Civil Engineering, Kaynaklar } \\
\text { Yerleşkesi, Buca, Izmir - TURKEY. } \\
\text { okan.onal@deu.edu.tr }\end{array}$ & $\begin{array}{l}\text { Turkish Journal of } \\
\text { Agriculture and } \\
\text { Forestry }\end{array}$ \\
\hline 27. & $\begin{array}{l}\text { SaadatKamgar and } \\
\text { Mohammad J. } \\
\text { Eslami }\end{array}$ & & $\begin{array}{l}\text { Design, Development and } \\
\text { Evaluation of a Mechatronic } \\
\text { Transmission System for } \\
\text { Upgrading Performance of a Row } \\
\text { Crop Planter }\end{array}$ & $\begin{array}{l}45 \mathrm{~W} \text { DC motor, tractor battery } \\
(12 \mathrm{~V} \& 75 \mathrm{Ah}), \text { microcontroller, MOSFET, } \\
\text { potentiometer, rotary shaft encoder } \\
\text { (E50S8-1000), } 4 \times 4 \text { matrix keyboard and } \\
2 \times 16 \mathrm{LCD}\end{array}$ & $\begin{array}{l}\text { Kamgar\&Eslami, } \\
\text { (2012) }\end{array}$ & $\begin{array}{l}\text { Agricultural Engineering Department, } \\
\text { Shiraz University, Shiraz, Iran. } \\
\text { kamgar@ shirazu.ac.ir }\end{array}$ & $\begin{array}{l}\text { American Society } \\
\text { of Agricultural } \\
\text { and Biological } \\
\text { Engineers }\end{array}$ \\
\hline 29. & $\begin{array}{l}\text { Qi Jiangtao, } \\
\text { JiaHonglei, Li Yang, } \\
\text { Yu Haibo, Liu } \\
\text { Xinhui, LanYubin, } \\
\text { FengXianzhen and } \\
\text { Yang Yongxi }\end{array}$ & Corn & $\begin{array}{l}\text { Design and Test of Fault } \\
\text { Monitoring System for Corn } \\
\text { Precision Planter }\end{array}$ & $\begin{array}{l}\text { Capacitive sensors (TAP-30D40N1-D3 } \\
\text { model), Display module } \\
\text { (JM160128BLCD), matrix keyboard, } \\
\text { single-chip microcomputer } \\
\text { (STC12C5A60S2), alarm module, input } \\
\text { module, count chip (74LS590), encoder } \\
\text { (PHB8-3600-G05L) and 8255A chip. }\end{array}$ & $\begin{array}{l}\text { Jiangtao et al., } \\
\text { (2015) }\end{array}$ & $\begin{array}{l}\text { Key Laboratory of Bionic Engineering } \\
\text { (Ministry of Education), Jilin } \\
\text { University, Changchun } 130022 \text {, China }\end{array}$ & $\begin{array}{l}\text { International } \\
\text { Journal of } \\
\text { Agricultural and } \\
\text { Biological } \\
\text { Engineering }\end{array}$ \\
\hline 30. & $\begin{array}{l}\text { Yang Li, He Xiantao, } \\
\text { Cui Tao, Zhang } \\
\text { Dongxing, Shi Song, } \\
\text { Zhang Rui and Wang } \\
\text { Mantao }\end{array}$ & Corn & $\begin{array}{l}\text { Development of Mechatronic } \\
\text { Driving System for Seed Meters } \\
\text { Equipped on Conventional } \\
\text { Precision Corn Planter }\end{array}$ & $\begin{array}{l}\text { Two-phase hybrid stepper motor } \\
\text { (57HBP76AL4-TF0), tractor battery, } \\
\text { driver module (2HD403), rotary shaft } \\
\text { encoder (TRD-2T500BF), touchscreen } \\
\text { (MT4414T), RS485 and microcontroller } \\
\text { (STM32F103VCT6) }\end{array}$ & Li et al., (2015) & $\begin{array}{l}\text { College of Engineering, China } \\
\text { Agricultural University, Beijing 100083, } \\
\text { China zhangdx@ cau.edu.cn }\end{array}$ & $\begin{array}{l}\text { International } \\
\text { Journal of } \\
\text { Agricultural and } \\
\text { Biological } \\
\text { Engineering }\end{array}$ \\
\hline 31. & $\begin{array}{l}\text { HabibKocabiyik, } \\
\text { Anil Cay, Bilal } \\
\text { Karaaslan, Sahin } \\
\text { May and M. } \\
\text { Khurelbaatar }\end{array}$ & Corn & $\begin{array}{l}\text { Electro-mechanic Control System } \\
\text { for Pneumatic Precision Corn } \\
\text { Planters }\end{array}$ & $\begin{array}{l}\text { ESC (electronic speed controller), driver } \\
\text { module, DC electric motors, encoders, } \\
\text { cables connectors ,PWM and PID } \\
\text { techniques }\end{array}$ & $\begin{array}{l}\text { Kocabiyik et al., } \\
\text { (2016) }\end{array}$ & $\begin{array}{l}\text { CanakkaleOnsekiz Mart University, } \\
\text { Faculty of Agriculture, Department of } \\
\text { Agricultural Machinery and } \\
\text { Technologies Engineering, 17020, } \\
\text { Canakkale, Turkey. }\end{array}$ & $\begin{array}{l}\text { International } \\
\text { Conference on } \\
\text { Machine Control } \\
\text { and Guidance }\end{array}$ \\
\hline
\end{tabular}




\begin{tabular}{|c|c|c|c|c|c|c|c|}
\hline & $\begin{array}{l}\text { Ajay Sharda, Daniel } \\
\text { Flippo, Ryan Strasser } \\
\text { and Terry Griffin }\end{array}$ & & $\begin{array}{l}\text { Camera Hardware and Software } \\
\text { Package to Evaluate Real-Time } \\
\text { Electric Seed Meter Accuracy of a } \\
\text { Variable Rate Planter }\end{array}$ & $\begin{array}{l}\text { speed sensors, control laptop computer, } \\
\text { Compact Rio (DAS), bore encoders, } \\
\text { controller display, seed tube Sensors and } \\
\text { high-speed camera }\end{array}$ & (2017) & $\begin{array}{l}\text { Engineering, Kansas State University, } \\
\text { 1016 Seaton Hall, Manhattan, KS } \\
\text { 66506, United States }\end{array}$ & $\begin{array}{l}\text { Electronics in } \\
\text { Agriculture }\end{array}$ \\
\hline 33. & $\begin{array}{l}\text { X. He, T. Cui, D. } \\
\text { Zhang, J. Wei, M. } \\
\text { Wang, Y. Yu, Q. Liu, } \\
\text { B. Yan, D. Zhao and } \\
\text { L. Yang }\end{array}$ & Corn & $\begin{array}{l}\text { Development of an Electric- } \\
\text { Driven Control System for a } \\
\text { Precision Planter Based on a } \\
\text { Closed-Loop PID Algorithm }\end{array}$ & $\begin{array}{l}\text { Display (MT4414T), incremental encoder } \\
\text { (TRD-2T500BF), four drive motors, 12V } \\
\text { tractor battery, Hall-effect sensors, Optical } \\
\text { Coupler (PC357), RS232 Transceiver } \\
\text { (MAX232), Main Controller } \\
\text { (STM32F103VCT6), Auxiliary Controller } \\
\text { (STM32F103RBT6) and power MOSFET }\end{array}$ & He et al., (2017) & $\begin{array}{l}\text { College of Engineering, China } \\
\text { Agricultural University, Beijing 100083, } \\
\text { China. yl_hb68@126.com }\end{array}$ & $\begin{array}{l}\text { Computers and } \\
\text { Electronics in } \\
\text { Agriculture }\end{array}$ \\
\hline 34. & $\begin{array}{l}\text { YongliangHao, Tao } \\
\text { Cui, Ganesh Bora, } \\
\text { Dongxing Zhang, } \\
\text { Jiantao Wei, Xiantao } \\
\text { He, Mantao Wang } \\
\text { and Li Yang }\end{array}$ & Corn & $\begin{array}{l}\text { Development of an Instrument to } \\
\text { Measure Planter Seed Meter } \\
\text { Performance }\end{array}$ & $\begin{array}{l}\text { Servo motor and gearbox, motor driver, } \\
\text { seed sensor, PLC controller (CPU504EX) } \\
\text {,tablet computer and printer (J625DW) }\end{array}$ & Hao et al., (2017) & $\begin{array}{l}\text { College of Engineering, China } \\
\text { Agricultural University, Beijing 100083, } \\
\text { China. } \\
\text { yangli@cau.edu.cn }\end{array}$ & $\begin{array}{l}\text { Applied } \\
\text { Engineering in } \\
\text { Agriculture }\end{array}$ \\
\hline 35. & $\begin{array}{l}\text { Anil Cay, } \\
\text { HabibKocabiyik and } \\
\text { Sahin May }\end{array}$ & Corn & $\begin{array}{l}\text { Development of an electro- } \\
\text { mechanic control system for seed- } \\
\text { metering unit of single seed corn } \\
\text { planters Part I: Design and } \\
\text { laboratory simulation }\end{array}$ & $\begin{array}{l}\text { Control panel, processor, electronic speed } \\
\text { controller, driver module, brushless DC } \\
\text { electric motors and other Supplementary } \\
\text { elements such as encoders, cables and } \\
\text { connectors. }\end{array}$ & Cay et al., (2018) & $\begin{array}{l}\text { Department of Agricultural Machinery } \\
\text { and Technologies Engineering, Faculty } \\
\text { of Agriculture, CanakkaleOnsekiz Mart } \\
\text { University, Canakkale, Turkey }\end{array}$ & $\begin{array}{l}\text { Computers and } \\
\text { Electronics in } \\
\text { Agriculture }\end{array}$ \\
\hline 36. & $\begin{array}{l}\text { Anil Cay, } \\
\text { HabibKocabiyik and } \\
\text { Sahin May }\end{array}$ & Corn & $\begin{array}{l}\text { Development of an Electro- } \\
\text { Mechanic Control System for } \\
\text { Seed-Metering Unit of Single } \\
\text { Seed Corn Planters Part II: Field } \\
\text { Performance }\end{array}$ & $\begin{array}{l}\text { Control panel, processor, electronic speed } \\
\text { controller, driver module, brushless DC } \\
\text { electric motors and other Supplementary } \\
\text { elements such as encoders, cables and } \\
\text { connectors }\end{array}$ & Cay et al., (2018) & $\begin{array}{l}\text { Department of Agricultural Machinery } \\
\text { and Technologies Engineering, Faculty } \\
\text { of Agriculture, CanakkaleOnsekiz Mart } \\
\text { University, Canakkale, Turkey }\end{array}$ & $\begin{array}{l}\text { Computers and } \\
\text { Electronics in } \\
\text { Agriculture }\end{array}$ \\
\hline
\end{tabular}

Table.4 Mechatronics in Magnetic Planter and Trans-Planter

\begin{tabular}{|c|c|c|c|c|c|c|c|}
\hline $\mathrm{S} / \mathrm{N}$ & Researcher Name & Seed & Research Topic & Electronic Components & Source & Address of Researcher & Journal Name \\
\hline 37. & $\begin{array}{l}\text { Yan Xiaoyue, Hu } \\
\text { Jianping, Ma Jun and } \\
\text { Wang Xun }\end{array}$ & Rape seeds & $\begin{array}{l}\text { Design of a Control System for } \\
\text { Magnetic Plate-type Precision } \\
\text { Seeding Production Line Based on } \\
\text { PLC and MCU. }\end{array}$ & $\begin{array}{l}\text { Permanent magnetic plugs, AC } \\
\text { motors(90YYJ (T) 120-30), controller( ES / } \\
\text { EX series Delta DVP-40ES PLC), step Motor } \\
\text { (110BF-003 and 85STH118), step motor } \\
\text { driver (BQH-300Y and WZM-2H057M), } \\
\text { LJD-51-XB +MCU, photoelectric sensors } \\
\text { (SICK GL6-N1111), capacitive proximity } \\
\text { sensors (SND05-N) and controller (DVP- } \\
\text { 40ES PLC) }\end{array}$ & $\begin{array}{l}\text { Xiaoyue et al., } \\
\text { (2013) }\end{array}$ & $\begin{array}{l}\text { Laboratory of Modern Agricultural } \\
\text { Equipment and Technology, Ministry of } \\
\text { Education and Jiangsu Province, Jiangsu } \\
\text { University, Zhenjiang, 212013, China }\end{array}$ & $\begin{array}{l}\text { Electrical and } \\
\text { Electronic } \\
\text { Engineering } \\
\text { Journal }\end{array}$ \\
\hline 38. & $\begin{array}{l}\text { Liguo Wei, Xiaochao } \\
\text { Zhang, QuanJia, and } \\
\text { Yangchun Liu }\end{array}$ & Rice & $\begin{array}{l}\text { Automatic Navigation System } \\
\text { Research for PZ60 Rice Planter }\end{array}$ & $\begin{array}{l}\text { RTK-GNSS receiver, electro-hydraulic } \\
\text { proportional valve, attitude transducer, fuzzy } \\
\text { control unit and engine power }(8.3 \mathrm{~kW})\end{array}$ & Wei et al., (2014) & $\begin{array}{l}\text { Chinese Academy of Agriculture } \\
\text { Mechanization Sciences, Beijing 100083, } \\
\text { China weilg78@126.com }\end{array}$ & $\begin{array}{l}\text { International } \\
\text { Conference on } \\
\text { CCTA }\end{array}$ \\
\hline 39. & $\begin{array}{l}\text { B. M. Nandede and } \\
\text { H. Raheman }\end{array}$ & $\begin{array}{l}\text { Pot seedlings of } \\
\text { tomato brinjal } \\
\text { and chilli }\end{array}$ & $\begin{array}{l}\text { Digital Seedling Counter for } \\
\text { Detection of Seedling Flow and } \\
\text { Spacing in Vegetable Trans planter: } \\
\text { A Low Cost Solution }\end{array}$ & $\begin{array}{l}\text { Microcontroller (PIC16F877A), LCD display } \\
\text { and RS232 port, power supply unit ( } 12 \mathrm{~V} \\
\text { batteries or } 220 \text { VAC supply), transformer, } \\
\text { rectifier diodes, regulator IC, capacitors, light } \\
\text { source, optical sensor, comparator and LED. }\end{array}$ & $\begin{array}{l}\text { Nandede\&Rahman, } \\
\text { (2016) }\end{array}$ & $\begin{array}{l}\text { ICAR-central Institute of Agricultural } \\
\text { Engineering, Bhopal, MP (India) }\end{array}$ & $\begin{array}{l}\text { International } \\
\text { Journal of } \\
\text { Engineering } \\
\text { Research and } \\
\text { Management } \\
\text { Technology }\end{array}$ \\
\hline
\end{tabular}


Table.5 Seed Sowing Robot

\begin{tabular}{|c|c|c|c|c|c|c|c|}
\hline $\mathrm{S} / \mathrm{N}$ & Researcher Name & Seed & Research Topic & Electronic Components & Source & Address of Researcher & Journal Name \\
\hline 40. & $\begin{array}{l}\text { M. Priyadarshini and L. } \\
\text { Sheela }\end{array}$ & & $\begin{array}{l}\text { Command based Self- } \\
\text { Guided Digging and Seed } \\
\text { Sowing Rover }\end{array}$ & $\begin{array}{l}\text { DC motor, Ultrasonic Radar sensor, sowing control } \\
\text { sensor, relay driver circuit, Wireless controller, PC, } \\
\text { Bluetooth module,vBattery package, Arduino mega2560 } \\
\text { microcontroller and LCD module }\end{array}$ & $\begin{array}{l}\text { Priyadarshini\&Sheela, } \\
\text { (2015) }\end{array}$ & $\begin{array}{l}\text { Dept of EEE, Embedded System } \\
\text { Technologies Regional office:Anna } \\
\text { University Tirunelveli, India }\end{array}$ & $\begin{array}{l}\text { International } \\
\text { Conference on } \\
\text { Engineering Trends } \\
\text { and Science and } \\
\text { Humanities }\end{array}$ \\
\hline 41. & $\begin{array}{l}\text { Swati D. Sambare and S. S. } \\
\text { Belsare }\end{array}$ & & $\begin{array}{l}\text { Use of robotics technology } \\
\text { for seed sowing in } \\
\text { Agriculture }\end{array}$ & $\begin{array}{l}\text { Keyboard, Zigbee module, PC, IR sensor, L293D driver } \\
\text { module, DC Motor, stepper motor, UNL2803, LCD, } \\
\text { LPC2148microcotrolle, MAC layers }\end{array}$ & $\begin{array}{l}\text { Sambare\&Belsare, } \\
(2015)\end{array}$ & $\begin{array}{l}\text { Dept. of Electronics, BVDUCOEP, } \\
\text { Pune, India } \\
\text { sambare.swati@gmail.com }\end{array}$ & IJSRM \\
\hline 42. & $\begin{array}{l}\text { Lin Haibo, Dong Shuliang, } \\
\text { Liu Zunmin and Yi Chuijie }\end{array}$ & Wheat & $\begin{array}{l}\text { Study and Experiment on } \\
\text { a Wheat Precision Seeding } \\
\text { Robot }\end{array}$ & $\begin{array}{l}\text { Drive motor, Steer motor, Seeding motor, Motor driver, } \\
\text { Motor controller, Controller, PC, Lead-acid } \\
\text { Batteries, sensor for pressure and speed }\end{array}$ & Haibo et al., (2015) & $\begin{array}{l}\text { College of Mechanical Engineering, } \\
\text { Qingdao Technological University, } \\
\text { Qingdao 266520, China }\end{array}$ & Journal of Robotics \\
\hline 43. & $\begin{array}{l}\text { Neha S. Naik, Virendra. V. } \\
\text { Shete and Shruti R. Danve }\end{array}$ & $\begin{array}{l}\text { Cotton, } \\
\text { Maize, } \\
\text { Soybean } \\
\text { and } \\
\text { Wheat }\end{array}$ & $\begin{array}{l}\text { Precision Agriculture } \\
\text { Robot for Seeding } \\
\text { Function }\end{array}$ & $\begin{array}{l}\text { Power supply( } 9 \text { and } 12 \mathrm{~V} \text { DC), input switches, IR } \\
\text { sensors, relays, 16x2 LCD display, DC motors, motor } \\
\text { driver (L293D IC), ARM7 board, microcontroller } \\
\text { (LPC2148) }\end{array}$ & Naik et al., (2016) & $\begin{array}{l}\text { Department of E and TC, MITCOE, } \\
\text { Pune, India } \\
\text { nhnk27@gmail.com }\end{array}$ & $\begin{array}{l}\text { International } \\
\text { Conference on IEEE }\end{array}$ \\
\hline 44. & $\begin{array}{l}\text { Kiran AS and } \\
\text { BabanParisaDathwade }\end{array}$ & & $\begin{array}{l}\text { Design and Fabrication of } \\
\text { Automatic Seed Sowing } \\
\text { Machine with Variable } \\
\text { Pitch }\end{array}$ & $\begin{array}{l}\text { Microcontroller, DC Gear Motor, rotary encoder, Battery, } \\
\text { Keypad }\end{array}$ & $\begin{array}{l}\text { Kiran\&Dathwade, } \\
\text { (2016) }\end{array}$ & $\begin{array}{l}\text { Department of Mechanical Engineering, } \\
\text { BCE, Shravanabelagola, India } \\
\text { kiranas.apri192@gmail.com }\end{array}$ & $\begin{array}{l}\text { European Journal of } \\
\text { Advances in } \\
\text { Engineering and } \\
\text { Technology }\end{array}$ \\
\hline 45. & $\begin{array}{l}\text { Palepu V. Santhi, } \\
\text { NelloreKapileswar, Vijay } \\
\text { K. R. Chenchela and } \\
\text { Venkata Siva Prasad. CH }\end{array}$ & & $\begin{array}{l}\text { Sensor and vision based } \\
\text { autonomous AGRIBOT } \\
\text { for sowing seeds }\end{array}$ & $\begin{array}{l}\text { Controller (Arduino), Ultrasonic and IR sensors, vision } \\
\text { sensor, power supply, PC }\end{array}$ & Santhi et al., (2017) & $\begin{array}{l}\text { Department of Electrical, Electronic and } \\
\text { Computer Engineering, University of } \\
\text { Pretoria, South Africa }\end{array}$ & ICECDS \\
\hline 46. & $\begin{array}{l}\text { AnujaMohalkar,PritiMohite, } \\
\text { ShubhangiNagare, and } \\
\text { SampadaTavse }\end{array}$ & & $\begin{array}{l}\text { Automatic Seed Sowing } \\
\text { Machine using Solar Panel }\end{array}$ & $\begin{array}{l}\text { Microcontroller (PIC16F877A), Keypad, LCD, DC Motor } \\
\text { Driver (L293D), IR Sensor, DC motors, } 12 \text { V battery, } \\
\text { amplifier, buzzer, keypad and solar panel }\end{array}$ & Mohalkar et al., 2017) & $\begin{array}{l}\text { Department of E and TC Engineering, } \\
\text { MarathwadaMitraMandal's College of } \\
\text { Engineering, Pune, India } \\
\text { anujamohalkar@gmail.com }\end{array}$ & $\begin{array}{l}\text { International Journal } \\
\text { of Innovations in } \\
\text { Engineering Research } \\
\text { and Technology }\end{array}$ \\
\hline 47. & RohanChauhan & & $\begin{array}{l}\text { Electronic Demarcation } \\
\text { Technique for Robotic } \\
\text { Precision Planter }\end{array}$ & $\begin{array}{l}\text { Microcontroller(Arduino Mega), DOF IMU, rotary } \\
\text { encoder, stepper motors, sensors, raspberry Pi3, Camera, } \\
\text { motor board, battery, power bank, DC-DC converter and } \\
\text { Ubuntu Mate }\end{array}$ & Chauhan, (2017) & $\begin{array}{l}\text { Kalinga Institute of Industrial } \\
\text { Technology, School of Computer } \\
\text { Engineering, Bhubaneswar, Odisha, } \\
\text { India. }\end{array}$ & $\begin{array}{l}\text { Journal of Engineering } \\
\text { and Applied Sciences }\end{array}$ \\
\hline 48. & $\begin{array}{l}\text { T. V. Pavan, R. Suresh, K. } \\
\text { R. Prakash, and C. } \\
\text { Mallikarjuna }\end{array}$ & $\begin{array}{l}\text { Green } \\
\text { gram }\end{array}$ & $\begin{array}{l}\text { Design and Development } \\
\text { of Agribot for Seeding }\end{array}$ & $\begin{array}{l}12 \mathrm{~V} \text { DC geared motors, Arduino Uno board, Atmega328 } \\
\text { microcontroller, motor driver (L298), Voltage } \\
\text { regulator785, lead acid battery, Ultrasonic sensor, }\end{array}$ & Pavan et al., (2017) & $\begin{array}{l}\text { Dept. Of Industrial Automation } \\
\text { Engineering, VTU PG Studies, Mysuru, } \\
\text { Karnataka, India }\end{array}$ & $\begin{array}{l}\text { International Research } \\
\text { Journal of Engineering } \\
\text { and Technology }\end{array}$ \\
\hline 49. & $\begin{array}{l}\text { ShraddhaMuley and Warsha } \\
\text { S. Kandlikar }\end{array}$ & $\begin{array}{l}\text { Soybean, } \\
\text { Jowar, } \\
\text { Wheat } \\
\text { and } \\
\text { Peanut }\end{array}$ & $\begin{array}{l}\text { Robotic Vehicle for Seed } \\
\text { Planting and Weeding } \\
\text { Applications }\end{array}$ & $\begin{array}{l}\text { ultrasonic sensor, keypad, LDR, LED, DC motors, motor } \\
\text { driver, Arduino Due Board, microcontroller (Atmel } \\
\text { SAM3X8E ARM Cortex-M3 CPU), SDA and SCL pins, } \\
\text { Due and AVR-based boards, Ultrasonic ranging module } \\
\text { HC - SR04 and L293D IC }\end{array}$ & $\begin{array}{l}\text { Muley\&Kandlikar, } \\
\text { (2017) }\end{array}$ & $\begin{array}{l}\text { Department of Electronics Design and } \\
\text { Technology, National Institute of } \\
\text { Electronics and Information } \\
\text { Technology, Dr. B.A.M. University } \\
\text { Campus, Aurangabad India }\end{array}$ & $\begin{array}{l}\text { International Journal } \\
\text { for Innovative } \\
\text { Research in Science } \\
\text { and Technolog y }\end{array}$ \\
\hline 50. & $\begin{array}{l}\text { Nikita Chame, } \\
\text { MamtaJadhav, Priyanka } \\
\text { Tele and Snehal P. Hon }\end{array}$ & & $\begin{array}{l}\text { Design and } \\
\text { Implementation of } \\
\text { Automatic Seed Sowing } \\
\text { Robot }\end{array}$ & $\begin{array}{l}12 \mathrm{~V} \text { battery, voltage regulator, PID controller, LDR } \\
\text { sensor, IR sensor, DC motors driver IC L293D, DC } \\
\text { motors, ADC(Atmega328p), Servo motor, Pulse Width } \\
\text { Modulation }\end{array}$ & Chame et al., (2018) & $\begin{array}{l}\text { Department of Electronics and } \\
\text { Telecommunication, PES MCOE, Pune, } \\
\text { India }\end{array}$ & $\begin{array}{l}\text { International Journal } \\
\text { of Research in } \\
\text { Engineering, Science } \\
\text { and Management }\end{array}$ \\
\hline
\end{tabular}


Table.6 Limit values of performance criteria for precision seeding (Cay et al., 2018)

\begin{tabular}{|l|l|l|l|}
\hline QFI (\%) & MI (\%) & MULT (\%) & Classification \\
\hline$>\mathbf{9 8 . 6}$ & $<0.7$ & $<0.7$ & Very good \\
\hline$>\mathbf{9 0 . 4 - 9 8 . 6}$ & $\geq 0.7$ to $<4.8$ & $\geq 0.7$ to $<4.8$ & Good \\
\hline $\mathbf{8 2 . 3}$ to $\leq \mathbf{9 0 . 4}$ & $\geq 04.8$ to $<7.7$ & $\geq 04.8$ to $<10$ & Moderate \\
\hline$<\mathbf{8 2 . 3}$ & $>7.7$ & $>10$ & Insufficient \\
\hline
\end{tabular}

Table.7 Results Obtained in Different Study with Mechatronics

\begin{tabular}{|c|c|c|c|c|c|}
\hline \multirow[b]{2}{*}{ Source } & \multirow[b]{2}{*}{$\begin{array}{l}\text { Speed } \\
\left(\mathrm{Kmh}^{-1}\right)\end{array}$} & \multicolumn{4}{|c|}{ Results } \\
\hline & & QFI (\%) & MULT (\%) & MI (\%) & PREC (\%) \\
\hline Cay et al., (2018) & $5-10$ & $2.91-95.36$ & $0-1.73$ & $4.45-97.09$ & $8.79-22.14$ \\
\hline $\begin{array}{l}\text { Mangus et al., } \\
(2017)\end{array}$ & $2.4-16.1$ & 98.45 & 0.2 & 0.8 & --- \\
\hline Cay et al., (2018) & $5-10$ & 90.63 & 0.94 & 8.44 & 17.63 \\
\hline $\begin{array}{l}\text { Taghinezhad et al., } \\
\text { (2013) }\end{array}$ & $0.9-3.6$ & $89.72-93.43$ & $2.52-7.23$ & $2.81-7.26$ & --- \\
\hline Jianbo et al., (2014) & $3.6-7.2$ & $85.83-95$ & $0-3.34$ & $5-10.83$ & --- \\
\hline $\begin{array}{l}\text { Jiangtao et al., } \\
\text { (2015) }\end{array}$ & 4.00 & $89.4-91.46$ & $2.44-3.86 \%$ & $5.28-9.11$ & --- \\
\hline $\begin{array}{l}\text { Xiaoyue et al., } \\
(2013)\end{array}$ & $0.52 \mathrm{rad} / \mathrm{s}$ & 90.28 & 4.69 & 5.04 & --- \\
\hline $\begin{array}{l}\text { Singh and Mane, } \\
\text { (2011) }\end{array}$ & $1-3.4$ & 100 & 0 & 0 & $1.84-7.34$ \\
\hline Önal\&Önal, (2009) & $1.8-7.2$ & 91.30 & 2.90 & 5.80 & --- \\
\hline He et al., (2017) & $8.6-13$ & $96.9-98.81$ & --- & $1.19-3.1$ & $14.38-16.04$ \\
\hline Hao et al., (2017) & $3-12$ & $95.1-98.1$ & $0.2-0.7$ & $1-4.2$ & --- \\
\hline $\begin{array}{l}\text { Kocabiyik et al., } \\
(\mathbf{2 0 1 6 )}\end{array}$ & $5-10$ & $31.73-97.18$ & $0-0.82$ & $2.45-69.27$ & $9.57-14.07$ \\
\hline Li et al., (2015) & $9-12$ & $89.93-94.23$ & --- & $2.49-5.03$ & $17.85-18.80$ \\
\hline
\end{tabular}

Fig.1 Principle of mechatronics metering mechanism (Jiangtao et al., 2015)

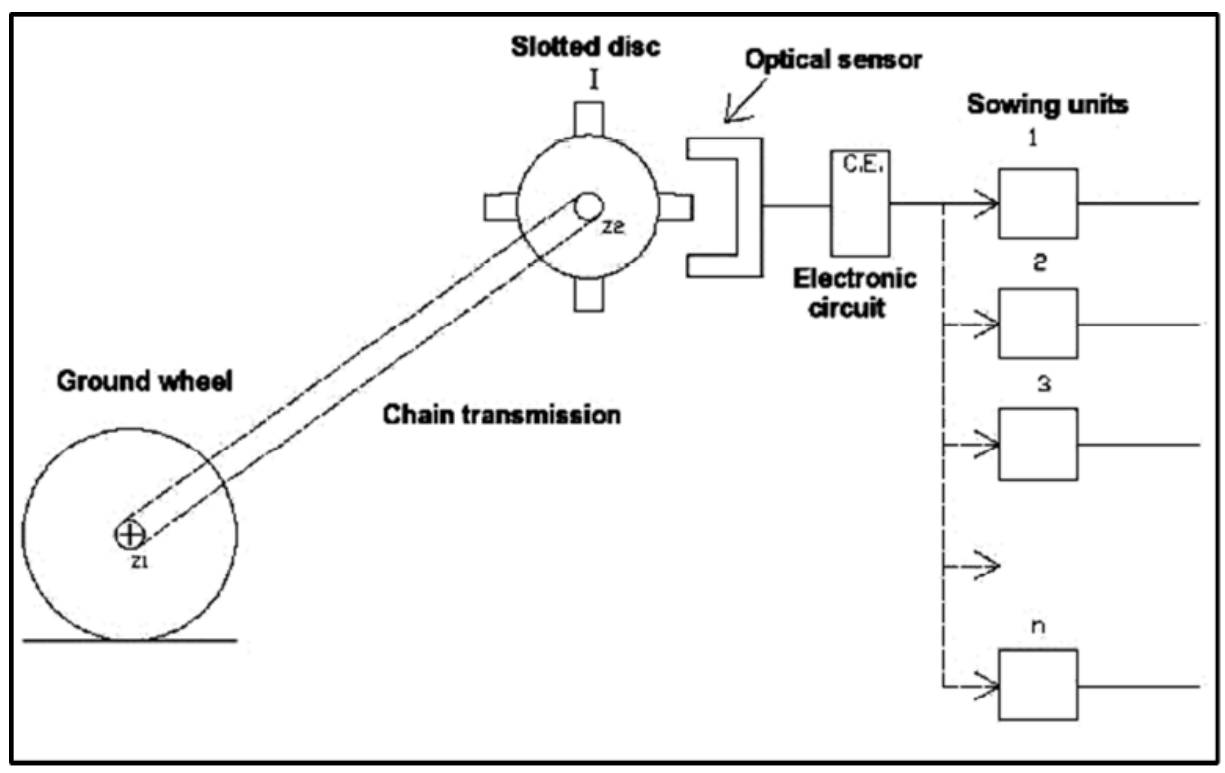


Fig.2 Components of the mechatronics drive system (He et al., 2017)

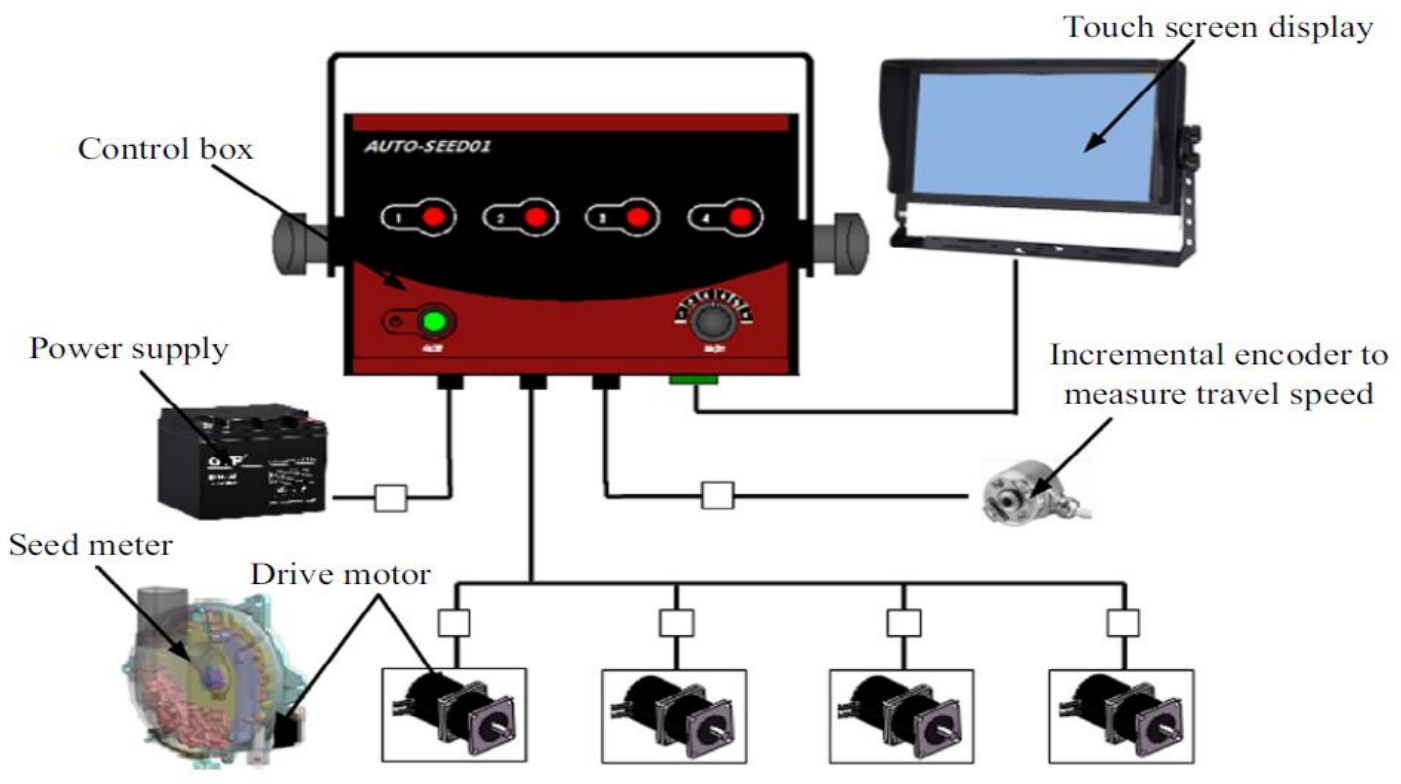

\section{Different studies' result}

It was observed that as the working speed increased, quality of feed index (QFI) of the seed-metering device dropped gradually with increased miss index (MI) and precision index (PREC) as well as deviation from the average seeds spacing became greater. The multiple index (MULT) decreases with increase in speed. The studies were indicated that precision planter equipped with mechatronics system observed good seeding uniformity among all seeding technologies with QFI, MI, MULT and PREC in range of 90-98, 0-11, 07 and 1-22 per cent, respectively under travel speed of 1 to $16 \mathrm{~km} \mathrm{~h}^{-1}$ (Table 7). Thus, mechatronics metering mechanism may be one of the options to achieve accurate seed spacing with higher efficiency in planting/seeding and capable to reduce the effect of higher speed of seeding.

It was observed that mechatronics driving system when attached with pneumatic plantercan reduce the effect of forward speed on planting accuracy effectively such as $4-8 \%$ increase in QFI and 4-7\%decrease in MI.
Mechatronics system solves the problems of existing precision planters like as ground wheel skidding.

Possibility of getting more transmission ratio by changing the pulse width modulation like as variable rate technology.

Good seeding uniformity and high productivity obtained because lowering precision index value of precision index up to $15 \%$.

Agribot gives near about 92\% accuracy regarding placement of different type of seeds and it can much more accurate in future.

\section{Acknowledgement}

With greatest honesty, I express my cordial gratitude to Division of Agricultural Engineering, IARI, New Delhi and CIAE, Bhopal for providing facilities during study.

\section{References}

Anantachar, M., Kumar, P. G., and Guruswamy, T. (2010).Neural network prediction of 
performance parameters of an inclined plate seed metering device and its reverse mapping for the determination of optimum design and operational parameters. Computers and Electronics in Agriculture, 72(2), 87-98.

Arnold, E. W., Brock, G. K., and Halley, D. G. (1979). U.S. Patent No. 4,141,390. Washington, DC: U.S. Patent and Trademark Office.

Aware, V. V., and Aware, S. V. (2014). Development of microprocessor based electronic metering mechanism for seedan approach. Engg. Tech. in India, 5(1\&2), 26-31.

Barut, Z. B., and YİĞİT, K (2008).Design of Electronic-Based Measurement System for Seed Spacing Measurement in Precision Planters.10th International Congress on Mechanization and Energy in Agriculture, 14-17 October 2008, Antalya-TURKIYE.

Cay, A., Kocabiyik, H., and May, S. (2018). Development of an electro-mechanic control system for seed-metering unit of single seed corn planters Part I: Design and laboratory simulation. Computers and Electronics in Agriculture, 144, 71-79.

Cay, A., Kocabiyik, H., and May, S. (2018). Development of an electro-mechanic control system for seed-metering unit of single seed corn planters Part II: Field performance. Computers and Electronics in Agriculture, 145, 11-17.

Chame, N., Jadhav, M., Tele, P., and Hon, S. P. (2018). Design and Implementation of Automatic Seed Sowing Robot. International Journal of Research in Engineering, Science and Management, 1(5).

Chaney, P. P., Parish, R. L. and Sistler F. E. (1986).Automatic control system for a sugarcane planter. Applied Engineering in Agriculture, 2(2): 51-54.

Chauhan, R. (2017). Electronic Demarcation Technique for Robotic Precision Planter. Journal of Engineering and Applied Sciences, 12(7), 1840-1845.

Cuhac, C., Virrankoski, R., Häninen, P.,
Elmusrati, M., Hööpakka, H., and Palomäki, H. (2012, December).Seed flow monitoring in wireless sensor networks. In 2nd Workshop on Wireless Sensor Systems (WoWSS2012).

Gautam, P. V. (2017). 'Development of microcontroller based seed metering mechanism for planting of pulse crops'. M. Tech. Thesis. IARI, New Delhi.

Haibo, L., Shuliang, D., Zunmin, L., and Chuijie, Y. (2015). Study and experiment on a wheat precision seeding robot. Journal of Robotics, 2015, 12.

Hajahmed, O., Tola, E., Al-Gaadi, K. A., and Kheiralla, A. F. (2011). Development of an Opto-Electronic Monitoring System for Crop Planter Seed Metering Unit. Middle-East Journal of Scientific Research, 8(4), 732-738.

Hao, Y., Cui, T., Bora, G., Zhang, D., Wei, J., He, X., and Yang, L. (2017).Development of an Instrument to Measure Planter Seed Meter Performance. Applied engineering in agriculture, 33(1), 31-40.

He, P. X., Yang, M. J. and Chen, Z. H. (2003). Study on photoelectric controlled precision seeder. Transactions of the CSAM, 34(1): 47-49.

He, X., Cui, T., Zhang, D., Wei, J., Wang, M., Yu, Y., and Yang, L. (2017).Development of an electric-driven control system for a precision planter based on a closed-loop PID algorithm. Computers and Electronics in Agriculture, 136: 184-192.

Iacomi, C., and Popescu, O. (2015).A new concept for seed precision planting. Agriculture and agricultural science procedia, 6, 38-43.

Jafari, M., Hemmat, A., and Sadeghi, M. (2010). Development and performance assessment of a DC electric variable-rate controller for use on grain drills. Computers and Electronics in Agriculture, 73(1), 56-65.

Jianbo, Z., Junfang, X., Yong, Z., and Shun, Z. (2014). Design and experimental study of the control system for precision seedmetering device. International Journal of 
Agricultural and Biological Engineering, 7(3), 13-18.

Jiangtao, Q., Honglei, J., Yang, L., Haibo, Y., Xinhui, L., Yubin, L., and Yongxi, Y. (2015). Design and test of fault monitoring system for corn precision planter. International Journal of Agricultural and Biological Engineering, 8(6), 13-19.

Kachman, S. D., and Smith, J. A. (1995). Alternative measures of accuracy in plant spacing for planters using single seed metering. Transactions of American Society of Agricultural Engineers, 38(2), 379-388.

Kamgar, S., and Eslami, M. J. (2012). Design, development and evaluation of a mechatronic transmission system for upgrading performance of a row crop planter. In 2012 Dallas, Texas, July 29August 1, 2012 (p. 1).American Society of Agricultural and Biological Engineers.

Kamgar, S., Eslami, M. J., and Maharlouie, M. M. (2013). Design, development and evaluation of a mechatronic transmission system to improve the performance of a conventional row crop planter. International Journal of Agronomy and Plant Production, 4(3), 480-487.

Kamgar, S., Noei-Khodabadi, F., and Shafaei, S. M. (2015). Design, development and field assessment of a controlled seed metering unit to be used in grain drills for direct seeding of wheat. Information Processing in Agriculture, 2(3-4), 169176.

Kang, N., Xianfa, F., Yangchun, L., Chengxu, L., and Yanwei, Y. (2017).Optimized design and performance evaluation of an electric cup-chain potato metering device. International Journal of Agricultural and Biological Engineering, 10(2), 36-43.

Karimi, H., Navid, H., and Mahmoudi, A. (2015).Online laboratory evaluation of seeding-machine application by an acoustic technique. Spanish Journal of Agricultural Research, 13(1), 0202.
Kaur, T., and Kumar, D. (2013).Design and development of calibration unit for precision planter. International Journal of Computer Science, Engineering and Applications, 3(3), 11.

Kiran, A. S., and Dathwade, B. P. (2016). Design and Fabrication of Automatic Seed Sowing Machine with Variable Pitch. European Journal of Advances in Engineering and Technology, 3(9), 50-54.

Kocabiyik, H., Cay, A., Karaaslan, B., May, S., and Khurelbaatar, M. (2016). Electromechanic Control System for Pneumatic Precision Corn Planters. International Conference on Machine Control and Guidance.

Koley, S., Bhatt, Y. C., Singh, G., Joshi, S., and Jain, H. K. (2017). Development of Electronic Metering Mechanism for Precision Planting of Seeds. Int. J. Curr. Microbiol. App. Sci, 6(8), 3481-3487.

Kushwaha, H. L., Sinha, J. P., Khura, T. K., Kushwaha, D. K., Ekka, U., Purushottam, M., and Singh, N. (2016).Status and Scope of Robotics in Agriculture. In International Conference on Emerging Technologies in Agricultural and Food Engineering (Vol. 12, p. 163).

Lan, Y., Kocher, M. F., and Smith, J. A. (1999). Opto-electronic sensor system for laboratory measurement of planter seed spacing with small seeds. Journal of Agricultural Engineering Research, 72, 119-127.

Li, J. F. and Gao, X. Y. (2007). Systematic design of the drills speed automatic monitor. Journal of Gansu Agricultural University, 2: 92-95.

Li, Y., Xiantao, H., Tao, C., Dongxing, Z., Song, S., Zhang, R., and Mantao, W. (2015). Development of mechatronic driving system for seed meters equipped on conventional precision corn planter. International Journal of Agricultural and Biological Engineering, 8(4), 1-9.

Mangus, D. L., Sharda, A., Flippo, D., Strasser, R., and Griffin, T. (2017).Development of high-speed camera hardware and software 
package to evaluate real-time electric seed meter accuracy of a variable rate planter. Computers and Electronics in Agriculture, 142, 314-325.

Moyer, J. R., Roman, E. S., Lindwall, C. W., and Blackshaw, R. E. (1994). Weed management in conservation tillage systems for wheat production in North and South America. Crop Protection, 13(4): 243-259.

Muley, S., and Kandlikar, W. S. (2017).Robotic Vehicle for Seed Planting and Weeding Applications. International Journal for Innovative Research in Science and Technology, 3(12).

Naik, N. S., Shete, V. V., and Danve, S. R. (2016, August). Precision agriculture robot for seeding function. In Inventive Computation Technologies (ICICT), International Conference on (Vol. 2, pp. 1-3).IEEE.

Nandede, B. M., and Raheman, H. (2016). Digital Seedling Counter for Detection of Seedling Flow and Spacing in Vegetable Transplanter: A Low Cost Solution. International Journal of Engineering Research and Management Technology, 3(1), 2348-4039.

Navid, H., Ebrahimian, S., and Gassemzadeh, H. R. (2011). Laboratory evaluation of seed metering device using image processing method. Australian journal of agricultural Engineering, 2(1), 1.

Nielsen, S. K., Munkholm, L. J., Lamandé, M., Nørremark, M., Edwards, G. T., and Green, O. (2018). Seed drill depth control system for precision seeding. Computers and Electronics in Agriculture, 144, 174180.

Önal, O., and Önal, I. (2009). Development of a computerized measurement system for inrow seed spacing accuracy. Turkish Journal of Agriculture and Forestry, 33(2), 99-109.

Onwubolu, G. (2005). Mechatronics: principles and applications. ButterworthHeinemann.

Pavan, T. V., Suresh, R., Prakash, K. R., and Mallikarjuna, C. (2017). Design and
Development of Agribot for Seeding. International Research Journal of Engineering and Technology, Volume: 04 Issue: 05.

Priyadarshini, M., and Sheela, L. (2015). Command based self guided digging and seed sowing rover. In IntConf on Engineering Trends and Science and Humanities (pp. 5-9).

Raheman, H., and Kumar, R. (2015).An embedded system for detecting seed flow in the delivery tube of a seed drill.In Proceeding of International Conference on Advances in Chemical, Biological and Environmental Engineering, Singapore (pp. 236-241).

Ruicheng, D., Bingcai, G., Ningning, L., Chenchen, W., Zidong, Y., and Mingjian, M. (2013). Design and experiment on intelligent fuzzy monitoring system for corn planters. International Journal of Agricultural and Biological Engineering, 6(3), 11-18.

Sambare, S. D., and Belsare, S. S. (2015). Seed sowing using robotics technology. International Journal of scientific research and management (IJSRM), 3(5), 2889-2892.

Santhi, P. V., Kapileswar, N., Chenchela, V. K., and Prasad, C. V. S. (2017, August). Sensor and vision based autonomous AGRIBOT for sowing seeds. In 2017 International Conference on Energy, Communication, Data Analytics and Soft Computing (ICECDS) (pp. 242245).IEEE.

Shinde, P. R., Lende, A. B., Rane, S. V., Nawale, S. A., Patwardhan, M. S., and Gharate, L. V. (2009). Development and functional test of electronic metering mechanism for bullock drawn Jyoti Multicrop planter. International Journal of Agriculture Environment and Biotechnology, 2(3), 305-309.

Singh, K., Agrawal, K. N., Dubey, A. K., and Chandra, M. P. (2012, November). Development of the controller based seed cum fertilizer drill. In Intelligent Systems Design and Applications (ISDA), 2012 
12th International Conference on (pp. 369-374). IEEE.

Singh, T. P., and Mane, D. M. (2011). Development and laboratory performance of an electronically controlled metering mechanism for okra seed. AMAAgricultural Mechanization in Asia Africa and Latin America, 42(2), 63.

Spoorthy, M. N., and Muralidhara, K. N. (2018). Microcontroller based seeding and plowing using sensor and Bluetooth technology. International Journal of Advance Research, Ideas and Innovations in Technology, 4(3), 923-925.

Taghinezhad, J., Alimardani, R., and Jafary, A. (2013). Design a capacitive sensor for rapid monitoring of seed rate of sugarcane planter. Agricultural Engineering International: CIGR Journal, 15(4), 2329.

Tang, Y. H. and Zhang, J. G. (2009) Research on the planting drive system based on a tractor front-wheel speed. Journal of Agricultural Mechanization Research, 3: 21-23.

Velandia, M., Buschermohle, M., Larson, J. A., Thompson, N. M., and Jernigan, B. M. (2013). The economics of automatic section control technology for planters: A case study of middle and west Tennessee farms. Computers and electronics in agriculture, 95, 1-10.

Wei, L., Zhang, X., Jia, Q., and Liu, Y. (2014, September).Automatic Navigation System Research for PZ60 Rice Planter. In International Conference on Computer and Computing Technologies in Agriculture (pp. 653-661).Springer, Cham.

Wilkins, D. E., and Lenker, D. H. (1981). A microprocessor-controlled planter. Transactions of the ASAE, 24(1), 2-0004.

Xia, L., Wang, X., Geng, D., and Zhang, Q. (2010, October). Performance monitoring system for precision planter based on MSP430-CT171. In International Conference on Computer and Computing Technologies in Agriculture (pp. 158165). Springer, Berlin, Heidelberg.

Xiaoyue, Y., Jianping, H., Jun, M., and Xun, W. (2013). Design of a Control System for Magnetic Plate-type Precision Seeding Production Line Based on PLC and MCU. Open Electrical and Electronic Engineering Journal, 7, 82-89.

Yang, L., Shi, S., Cui, T., Zhang, D. X. and Gao, N. N. (2012). Air-suction corn precision metering device with mechanical supporting plate to assist carrying seed. Transactions of the CSAM, 43(s1): 48-53.

Yang, M. J., Yang, L. and He P. X. (2002). Development of a precision seeder control system. Journal of Southwest Agricultural University, 24(3): 286-288.

Zhai, J. B., Gao H. Z. and Zheng, X. L. (2011). Research on automatical seed metering drive system based on sensor technology. Hubei Agricultural Sciences, 50(17): 3619-3621, 3646.

\section{How to cite this article:}

Prem Veer Gautam, H.L. Kushwaha, Adarsh Kumar and Dilip Kumar Kushwaha. 2019. Mechatronics Application in Precision Sowing: A Review. Int.J.Curr.Microbiol.App.Sci. 8(04): 1793-1807. doi: https://doi.org/10.20546/ijcmas.2019.804.208 